Subscriber access provided by Caltech Library

\title{
Communication
}

\section{Phase Modulation with Electrically Tunable Vanadium Dioxide Phase-Change Metasurfaces}

Yonghwi Kim, Pin Chieh Wu, Ruzan Sokhoyan, Kelly A. Mauser, Rebecca Glaudell, Ghazaleh Kafaie Shirmanesh, and Harry A Atwater

Nano Lett., Just Accepted Manuscript • DOI: 10.1021/acs.nanolett.9b01246 • Publication Date (Web): 28 May 2019

Downloaded from http://pubs.acs.org on May 30, 2019

\section{Just Accepted}

"Just Accepted" manuscripts have been peer-reviewed and accepted for publication. They are posted online prior to technical editing, formatting for publication and author proofing. The American Chemical Society provides "Just Accepted" as a service to the research community to expedite the dissemination of scientific material as soon as possible after acceptance. "Just Accepted" manuscripts appear in full in PDF format accompanied by an HTML abstract. "Just Accepted" manuscripts have been fully peer reviewed, but should not be considered the official version of record. They are citable by the Digital Object Identifier (DOI®). "Just Accepted" is an optional service offered to authors. Therefore, the "Just Accepted" Web site may not include all articles that will be published in the journal. After a manuscript is technically edited and formatted, it will be removed from the "Just Accepted" Web site and published as an ASAP article. Note that technical editing may introduce minor changes to the manuscript text and/or graphics which could affect content, and all legal disclaimers and ethical guidelines that apply to the journal pertain. ACS cannot be held responsible for errors or consequences arising from the use of information contained in these "Just Accepted" manuscripts. 


\title{
Phase Modulation with Electrically Tunable Vanadium Dioxide Phase-Change Metasurfaces
}

\author{
Yonghwi Kim, ${ }^{\dagger}$ Pin Chieh Wu,${ }^{\dagger}$ Ruzan Sokhoyan,${ }^{\dagger}$ Kelly Mauser, ${ }^{\dagger}$ Rebecca Glaudell, ${ }^{\dagger}$ Ghazaleh \\ Kafaie Shirmanesh, ${ }^{\dagger}$ and Harry A. Atwater ${ }^{* \dagger}$ \\ †Thomas J. Watson Laboratories of Applied Physics, California Institute of Technology, Pasadena, California 91125, \\ United States \\ \#avli Nanoscience Institute, California Institute of Technology, Pasadena, California 91125, United States \\ *Corresponding author: Harry A. Atwater (haa@caltech.edu)
}

\begin{abstract}
We report a dynamically tunable reflectarray metasurface that continuously modulates the phase of reflected light in the near-infrared wavelength range under active electrical control of the phase transition from semiconducting to semimetallic states. We integrate a vanadium dioxide $\left(\mathrm{VO}_{2}\right)$ active layer into the dielectric gap of antenna elements in a reflectarray metasurface, which undergoes an insulator-to-metal transition upon resistive heating of the metallic patch antenna. The induced phase transition in the $\mathrm{VO}_{2}$ film strongly perturbs the magnetic dipole resonance supported by the metasurface. By carefully controlling the volume fractions of coexisting metallic and dielectric regions of the $\mathrm{VO}_{2}$ film, we observe a continuous shift of the phase of the reflected light, with a maximal achievable phase shift as high as $250^{\circ}$. We also observe a reflectance modulation of $23.5 \%$ as well as a spectral shift of the resonance position by $175 \mathrm{~nm}$. The metasurface phase modulation is fairly broadband, yielding large phase shifts at multiple operation wavelengths.
\end{abstract}

KEYWORDS: Metasurface, reflectarray, vanadium dioxide, phase modulation, near-infrared

Optical metasurfaces are judiciously designed arrays of subwavelength optical resonators, which interact with incident light and alter the properties of the scattered electromagnetic waves, such as amplitude, phase, wavelength, and polarization. ${ }^{1,2}$ Optical metasurfaces have drawn tremendous attention due to their promise in replacing conventional bulky optical components with low-profile nanophotonic analogs. Moreover, a single metasurface may realize an optical function which otherwise can only be attained by combining multiple bulky optical components. ${ }^{3}$ While bulky optical components tailor the wavefront of the scattered light via phase accumulation which occurs when electromagnetic waves propagate through the given medium, metasurfaces utilize 
subwavelength optical scatterers, which tailor the wavefront of the scattered light by introducing abrupt changes in the properties of the scattered light at subwavelength scale. Metasurfaces have been designed to demonstrate a number of optical components such as anomalous reflectors, ${ }^{4,5}$ focusing lenses/mirrors, ${ }^{6,7}$ polarization convertors, ${ }^{3,8,9}$ and holographic plates. ${ }^{10-12}$ However, these metasurfaces are passive, so their optical response cannot be dynamically changed after fabrication.

The desire to dynamically control the key constitutive properties of light at subwavelength scale has given rise to a burgeoning field of active metasurfaces. While there are numerous reports of active amplitude control for scattered light, experimental demonstrations of dynamic phase control are rare. Both phase and amplitude control are required to achieve many important applications for dynamically tunable metasurfaces, such as chip-scale beam steering devices for light detection and ranging (LiDAR) systems, reconfigurable metalenses, and 3D holographic displays. The optical response of active metasurfaces can be dynamically changed upon application of external stimuli such as heat or electrical bias. Previous research has created active metasurfaces by employing a number of physical phenomena such as thermo-optic effects in semiconductors; ${ }^{13-15}$ field effect in indium tin oxide, ${ }^{16-20}$ gallium arsenide, ${ }^{21}$ silicon, ${ }^{22}$ and graphene, ${ }^{23-28}$ phase transitions in germanium antimony telluride (GST) ${ }^{29-34}$ and vanadium dioxide $\left(\mathrm{VO}_{2}\right) ;{ }^{35-44}$ as well as reorientation of liquid crystal molecules, ${ }^{45-48}$ ionic transport, ${ }^{49}$ and mechanical deformations. ${ }^{50-52}$ A grand challenge for the field of active metasurfaces is the realization of comprehensive active control of both amplitude and phase of the scattered electromagnetic waves. However, most reports of active control have demonstrated amplitude modulation whereas experimental demonstrations of dynamical control of the phase of electromagnetic waves are much less common. ${ }^{15,18-20,27,29,31-33,39,44,47,52}$ Continuous active control of phase variation of the scattered light throughout the spatial extent of a metasurface would enable complex wavefront engineering, enabling the manipulation of the properties of the scattered light. For example, prior research has reported a metasurface-based dynamic phase grating by temporally varying the spatial phase profile of the electromagnetic waves reflected from the metasurface. ${ }^{19}$ This enabled demonstration of electrical bias-actuated dynamic beam switching. Hence, from the point of view of future potential application, electrically tunable metasurfaces are especially interesting since they enable individual addressability to metasurface elements, holding promise to realize complex wavefront control. 
In this work, we demonstrate an electrically tunable metasurface, which enables an actively controllable phase shift of the scattered electromagnetic waves in the near-infrared wavelength range. To achieve dynamic metasurface tunability, we employ $\mathrm{VO}_{2}$ as an active material, whose optical properties can be changed upon application of an external stimulus. We integrate this active material into an otherwise passive metasurface structure and study the dynamically tunable optical response of the metasurface. Vanadium dioxide is a well-known phase change material, which undergoes a reversible insulator-to-metal transition upon heating. ${ }^{53}$ The phase transition of $\mathrm{VO}_{2}$ is accompanied by a large change in complex refractive index over a broad spectral range. The insulator-to-metal transition in $\mathrm{VO}_{2}$ occurs at a temperature of $T_{\mathrm{c}} \approx 340 \mathrm{~K},{ }^{54}$ which is close to room temperature. This makes $\mathrm{VO}_{2}$ a promising candidate for energy-efficient devices. While $\mathrm{VO}_{2}$ based tunable metasurfaces ${ }^{35-44}$ have been previously demonstrated, in the majority of these works, the insulator-to-metal transition in $\mathrm{VO}_{2}$ was induced either by direct heating ${ }^{29-31,35-39}$ or optical pumping. ${ }^{32,33,40}$ A limited number of works have reported electrically tunable $\mathrm{VO}_{2}$-based metasurfaces operating at near-,${ }^{43}$ mid-,${ }^{42}$ or far-infrared ${ }^{41}$ wavelengths. Although the electrical tuning mechanism is still induced by heating, the electrical controllability is essential to realize devices with complex functionalities such as phased array systems for beam steering applications, which require individual control of metasurface elements. ${ }^{19}$ These works, however, report only the intensity modulation of the scattered light and do not demonstrate phase modulation at optical frequencies. While a prior work has shown an electrically tunable phase of electromagnetic waves transmitted through the $\mathrm{VO}_{2}$-based metasurface, the operation wavelength is on the order of millimeters. ${ }^{44}$ The applications for the metasurface in our work, such as LiDAR or holographic displays, require the metasurface operation wavelength to be in the near-infrared wavelength ranges. It is worth mentioning that besides $\mathrm{VO}_{2}$, previous works have used amorphous-tocrystalline switching in GST to demonstrate active phase change metasurfaces. ${ }^{29-34}$ While a number of works on $\mathrm{VO}_{2}{ }^{-39,44}$ or GST-based ${ }^{29,31-33}$ active metasurfaces have benefited from the phase difference of the scattered light, an actively controlled continuous phase shift of nearinfrared electromagnetic waves scattered by $\mathrm{VO}_{2}$ - or GST-based active metasurfaces has not been demonstrated.

Here, we propose and experimentally demonstrate an electrically tunable $\mathrm{VO}_{2}$-based reflectarray metasurface, which exhibits an actively controlled wide phase shift in the near-infrared wavelength range. In our work, we incorporate $\mathrm{VO}_{2}$ into the dielectric gap of the reflectarray 
metasurface and actively induce the insulator-to-metal transition by flowing current through the top metal patch to resistively heat the $\mathrm{VO}_{2}$ (Figure 1a). By changing applied bias voltage, we finely control the temperature of the $\mathrm{VO}_{2}$ layer, inducing a partial insulator-to-metal transition in the $\mathrm{VO}_{2}$ layer. The electrical bias enables controlled and localized heating of $\mathrm{VO}_{2}$, which results in a continuous phase shift of the reflected light ranging from $0^{\circ}$ to $180^{\circ}$ at a wavelength of $1550 \mathrm{~nm}$. Moreover, we measure an actively controlled large phase shift over a remarkably broad wavelength range from 1515 to $1575 \mathrm{~nm}$. We measure a maximal phase shift of $250^{\circ}$ at a wavelength of $1520 \mathrm{~nm}$, which is close to the resonant wavelength of the metasurface when $\mathrm{VO}_{2}$ in a pure insulating state. The largest reflectance modulation of $23.5 \%$ occurs at a wavelength of $1680 \mathrm{~nm}$, corresponding to the resonant wavelength of the metasurface when $\mathrm{VO}_{2}$ is in a pure metallic state. Additionally, we observe that when $\mathrm{VO}_{2}$ undergoes an insulator-to-metal transition, the spectral position of the resonance dip shifts by $175 \mathrm{~nm}$. 

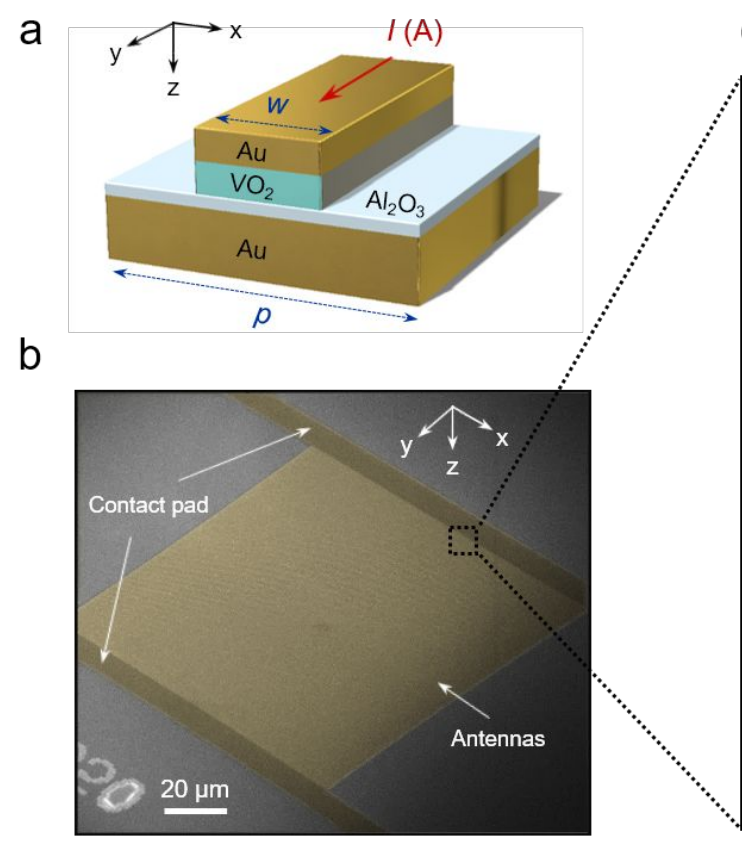

C

Figure 1. Electrically tunable $\mathrm{VO}_{2}$ metasurface. (a) Schematic illustration of a $\mathrm{VO}_{2}$-based tunable metasurface unit cell consisting of a metal-insulator-metal (MIM) structure. The metasurface device consists of a 40-nm-thick patterned Au stripe atop a 40-nm-thick active $\mathrm{VO}_{2}$ stripe, a 50-nm-thick $\mathrm{Al}_{2} \mathrm{O}_{3}$ layer, and an optically thick $(150 \mathrm{~nm})$ Au backplane. The width $(w)$ and the period $(p)$ of the unit cell of the metasurface are $210 \mathrm{~nm}$ and $400 \mathrm{~nm}$, respectively. (b) Scanning electron microscope (SEM) image (false color) of the metasurface consists of the MIM stripe antenna and the contact pad and (c) the close-up SEM image of the MIM stripe antenna and the contact pad. The top Au stripe simultaneously supports optical resonances and acts as a Joule heater via connection to external circuitry.

The unisssst cell of the designed metasurface is based on a metal-insulator-metal (MIM) structure as shown in Figure 1a. The phase transition in $\mathrm{VO}_{2}$ is thermally induced via Joule heating of the top Au stripe using an external current source. In this way the temperature of the $\mathrm{VO}_{2}$ can be carefully controlled. We incorporate the active material $\left(\mathrm{VO}_{2}\right)$ directly into the MIM structure to enhance the interaction between the active medium and the strongly confined field. The strong light-matter interaction in the resonance cavity of the metasurface results in large phase shifts of the reflected light induced by the significant change in the resonance of the unit cell. Furthermore, continuous phase shifts can be obtained by utilizing phase coexistence of the $\mathrm{VO}_{2}$ thin film ${ }^{54}$ via gradual Joule heating, which provides intermediate optical properties near the insulator-to-metal transition.

To demonstrate this modulation mechanism, we fabricated subwavelength metallic antenna array structures incorporating the $\mathrm{VO}_{2}$ active layer (Supporting Information, Part 1). A scanning 
electron microscope (SEM) image of the fabricated device is shown in Figure 1b. The large contact pads allow for uniform electrical connectivity to tune device response, and are wire-bonded to a chip carrier and circuit board for electrical control. The contact pads and subwavelength antennas composed of $\mathrm{Au}$ are false-colored yellow, while the grey region corresponds to the etched region on a fused silica substrate. The thicknesses of the layers within the stripe antenna, $\mathrm{Au}$ and $\mathrm{VO}_{2}$, are both $40 \mathrm{~nm}$. The 50 -nm-thick $\mathrm{Al}_{2} \mathrm{O}_{3}$ layer is deposited on an optically-thick Au backplane. The width and the period of the MIM antenna are $210 \mathrm{~nm}$ and $400 \mathrm{~nm}$, respectively. The total area of the antenna array is about $100 \mu \mathrm{m} \times 100 \mu \mathrm{m}$, which is larger than the incident beam spot size. The zoomed-in SEM image of the device (Figure 1c) shows the connection between the stripe antennas and contact pads in greater detail.

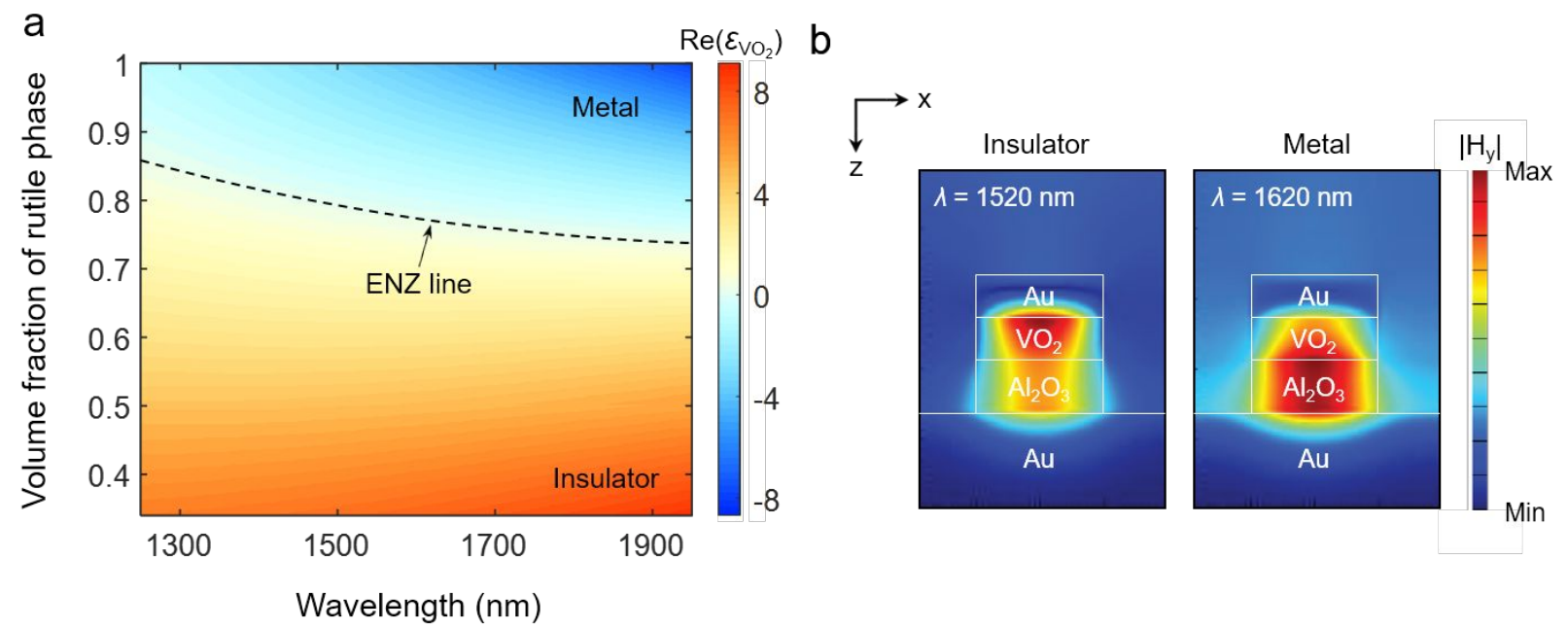

C

d
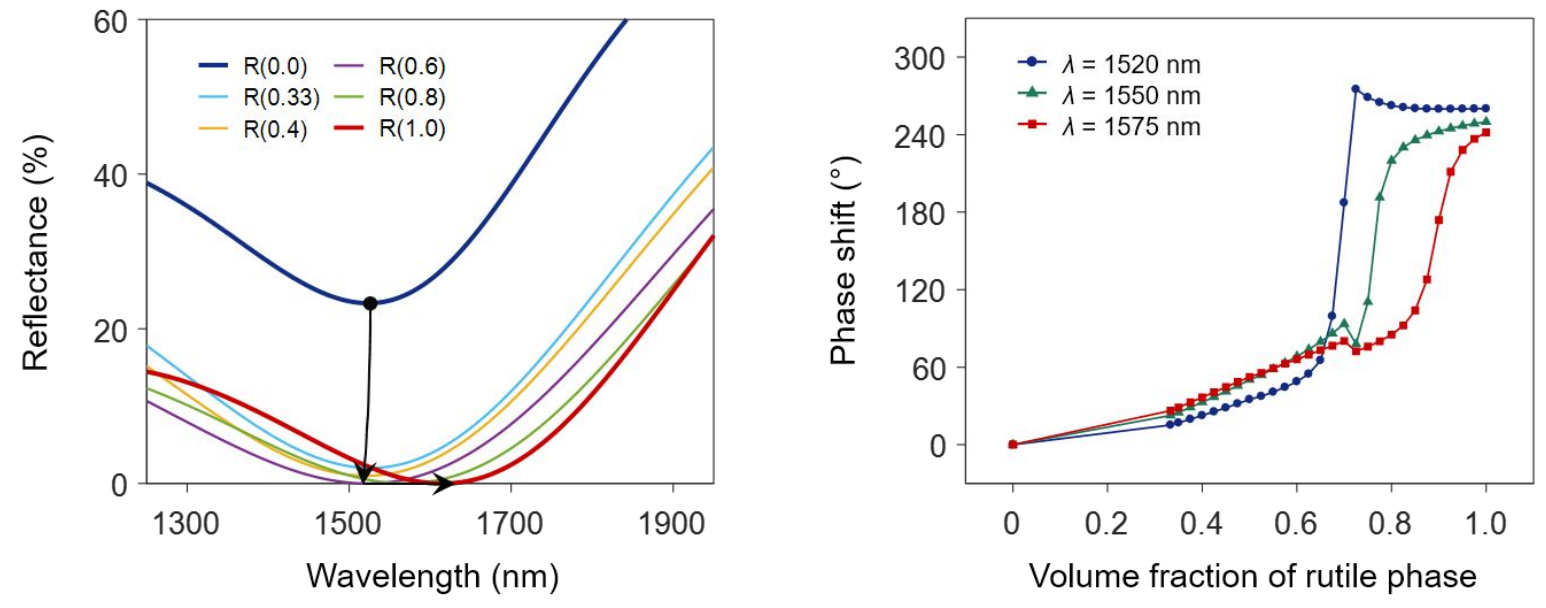
Figure 2. Simulation results of the active metasurface structure. (a) Real part of the dielectric permittivity of $\mathrm{VO}_{2}$ as a function of rutile phase volume fraction. The intermediate dielectric permittivity is estimated based on the Bruggeman effective medium approximation. The dashed curve indicates the region in which the real part of the dielectric permittivity of $\mathrm{VO}_{2}$ is zero. The epsilon-near-zero (ENZ) region implies an abrupt change in the optical properties of $\mathrm{VO}_{2}$, as the crystal structure changes from the insulating phase to the metallic phase. (b) Magnetic field magnitude in the metasurface unit cell, with $\mathrm{VO}_{2}$ in the insulating phase (left), and in the metallic phase (right) under normal incidence at each resonance wavelength. The incident illumination is TM-polarized (H-field vector parallel to the stripes). (c) Reflectance spectra for different volume fractions of the rutile phase in the $\mathrm{VO}_{2}$ layer. The legend $\mathrm{R}(0.0)$ corresponds to the case when $\mathrm{VO}_{2}$ is in the purely insulating phase, while the legend $\mathrm{R}(1.0)$ corresponds to the case when $\mathrm{VO}_{2}$ is in the purely metallic phase. (d) Phase modulation as a function of the rutile phase volume fraction in the $\mathrm{VO}_{2}$ layer for three different wavelengths, $\lambda=1520,1550$, and $1575 \mathrm{~nm}$.

A thin $\mathrm{VO}_{2}$ film consists of grains with a thermal hysteresis near the phase transition threshold temperature where the insulating and the metallic phases coexist. The characteristics of thermal hysteresis loop such as the threshold temperature and the hysteresis width show dependence on grain size and crystallinity, which can be affected by growth conditions and substrates. ${ }^{55,56}$ The phase transition in $\mathrm{VO}_{2}$ accompanies structural deformation from a monoclinic insulating phase to a tetragonal rutile metallic phase upon application of external stimuli. The insulator-to-metal transition in $\mathrm{VO}_{2}$ is initiated by generation of nanoscale metallic islands at randomly located nucleation sites followed by growth of these islands and connection via a percolation process. ${ }^{54,57-59}$ The spatial inhomogeneity induced by the coexistence of different phases yields an averaged intermediate dielectric permittivity of the $\mathrm{VO}_{2}$ film. In our tunable metasurface, this continuous modulation of the dielectric permittivity of $\mathrm{VO}_{2}$ can be obtained by controlling temperature via modulation of the voltage applied to the Au stripes. This mechanism allows us to control the temperature of the metasurface more finely than is possible by directional heating or laser heating, which is essential for future applications that require individual control of each of the metasurface antenna elements. In simulation (Figure 2a), we employed the Bruggeman effective medium approximation (EMA) to model the intermediate dielectric permittivity of $\mathrm{VO}_{2}$. The Bruggeman EMA was chosen as it can be applied to composites in which the inclusions cover a large range of volume fractions. ${ }^{60,61}$ We assumed the inclusion of rutile phase $\mathrm{VO}_{2}$ in the monoclinic phase $\mathrm{VO}_{2}$ medium and applied the Bruggeman EMA for rutile volume fractions of 0.33 or greater, which is the percolation threshold of insulator-to-metal transitions predicted from the Bruggeman EMA. The optical constants of insulating and metallic $\mathrm{VO}_{2}$ used in the Bruggeman 
EMA were obtained by spectroscopic ellipsometry measurements from a $\mathrm{VO}_{2}$ film grown on a sapphire substrate (Supporting Information, Part 5). The real part of the effective dielectric permittivity of $\mathrm{VO}_{2}$ modeled by the Bruggeman EMA is shown in Figure 2a. This illustrates that the real part of the dielectric permittivity of $\mathrm{VO}_{2}$ continuously decreases as the volume fraction of metallic inclusion increases. The change in sign of the permittivity at the epsilon-near-zero (ENZ) region (dashed line in Figure 2a) is indicative of the change in averaged optical properties of $\mathrm{VO}_{2}$ from insulating to metallic. For example, at a wavelength of $\lambda=1550 \mathrm{~nm}$, when the rutile fraction is larger than $\mathrm{R}(0.775)$, the $\mathrm{VO}_{2}$ layer possesses negative effective permittivity and therefore optically functions as a metal.

Based on the predicted effective permittivity of $\mathrm{VO}_{2}$, we performed full-wave electromagnetic simulations to understand the near- and the far-field characteristics of our $\mathrm{VO}_{2}$ based reflectarray metasurface. In the simulation results shown in Figure $2 b-d$, the optical response of the $\mathrm{VO}_{2}$ metasurface unit cell was characterized at normal incidence under transverse magnetic (TM) wave excitation. When $\mathrm{VO}_{2}$ is in the insulating phase, the incident plane wave excites a magnetic dipole resonance in the near-infrared, which is $\lambda=1520 \mathrm{~nm}$. As seen in the left panel of Figure $1 \mathrm{~b}$, the magnetic field is concentrated between the back reflector and top metallic stripe. Alternately, when the $\mathrm{VO}_{2}$ layer is in the metallic phase, the magnetic field is mainly confined in the $\mathrm{Al}_{2} \mathrm{O}_{3}$ layer as the effective thickness of the dielectric layer $\left(\mathrm{Al}_{2} \mathrm{O}_{3}\right.$ and $\left.\mathrm{VO}_{2}\right)$ decreases (the right panel of Figure 2b). The change in the near-field characteristics of the supported mode is accompanied by large changes in the amplitude and the phase of the reflected light. Figure $2 \mathrm{c}$ shows the change in the reflectance spectra of the metasurface as a function of the rutile volume fraction of $\mathrm{VO}_{2}$ phase. In the figure, $\mathrm{R}(0.0)$ and $\mathrm{R}(1.0)$ denote purely insulating phase and purely metallic phase, respectively. The values in between the two pure phases indicate intermediate phases defined by the volume fraction of the rutile phase. As the fraction of the rutile phase increases, the reflectance around the resonance dip decreases as $\mathrm{VO}_{2}$ becomes a lossier dielectric, and the dip reaches its minimum at $\approx \mathrm{R}(0.6)$. When the rutile phase fraction becomes the dominant phase $(\mathrm{R}>0.6)$, the resonance dip redshifts toward the wavelength at which the real part of the effective permittivity approches the ENZ region as shown in Figure 2a. The redshift of the resonance amounts to $\approx 100 \mathrm{~nm}$ when the $\mathrm{VO}_{2}$ layer crosses the $\mathrm{ENZ}$ region and transforms into the purely rutile phase. This redshift is expected as the effective thickness of the dielectric layer shrinks when the $\mathrm{VO}_{2}$ layer transforms into the metallic phase. ${ }^{19,20}$ Our simulations show that at 
the wavelength of the resonance dip in the insulating phase $(\lambda=1520 \mathrm{~nm})$ the reflectance from the metasurface decreases by $23.3 \%$. On the other hand, at the wavelength of the resonance dip in the metallic phase $(\lambda=1620 \mathrm{~nm})$ the reflectance decreases by $27.9 \%$.

Along with the amplitude modulation upon phase transition, full-wave simulations also predict a continuous phase modulation of the reflected light that can be achieved via fine control of the ratio of the coexisting phases. As shown in Figure 2d, a gradual change in the phase of scattered light can be obtained as the rutile volume fraction of $\mathrm{VO}_{2}$ increases. In contrast, we observe a larger change in the phase as it approches and crosses the ENZ region. For example, at $>\mathrm{R}(0.75)$ and a wavelength of $\lambda=1550 \mathrm{~nm}$, the reflectance dip experiences a redshift. Furthermore, our simulations show that the designed structure exhibits a significant phase shift of $\approx 260^{\circ}$ near the insulating-phase resonance wavelength $(\lambda=1520 \mathrm{~nm})$ where the phase of $\mathrm{VO}_{2}$ changes from purely insulating to purely metallic.

a

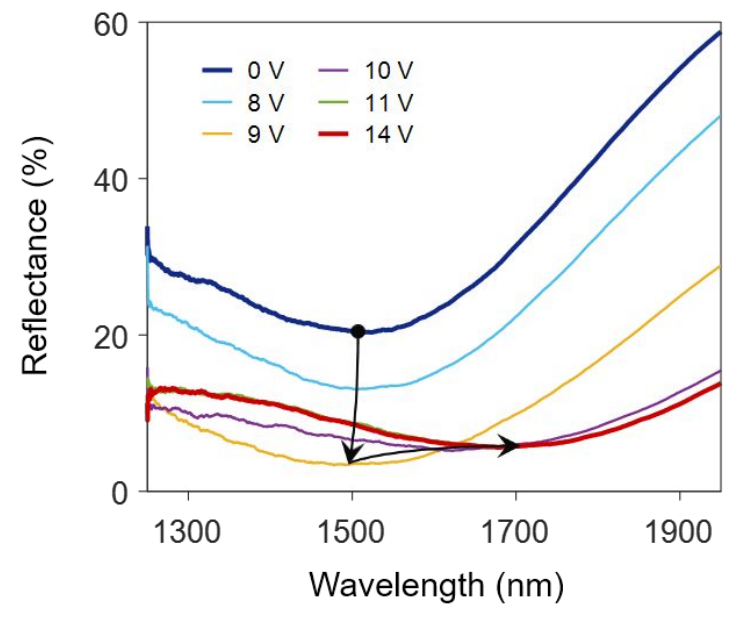

C

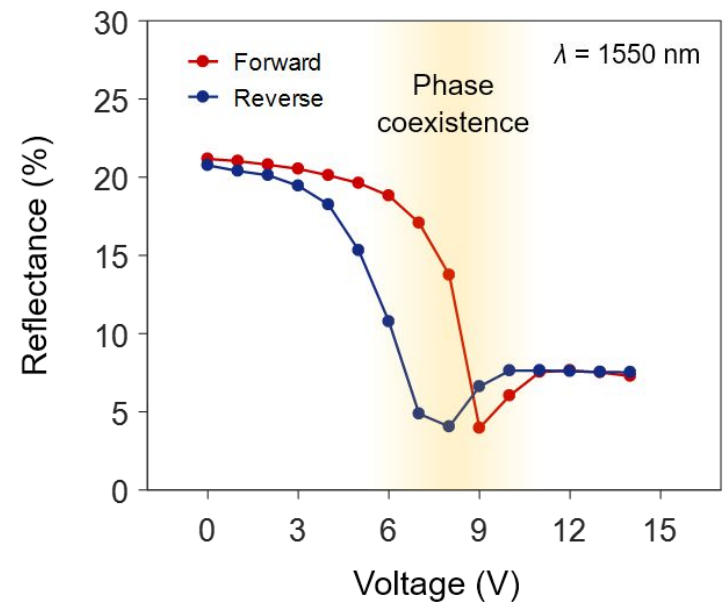

b

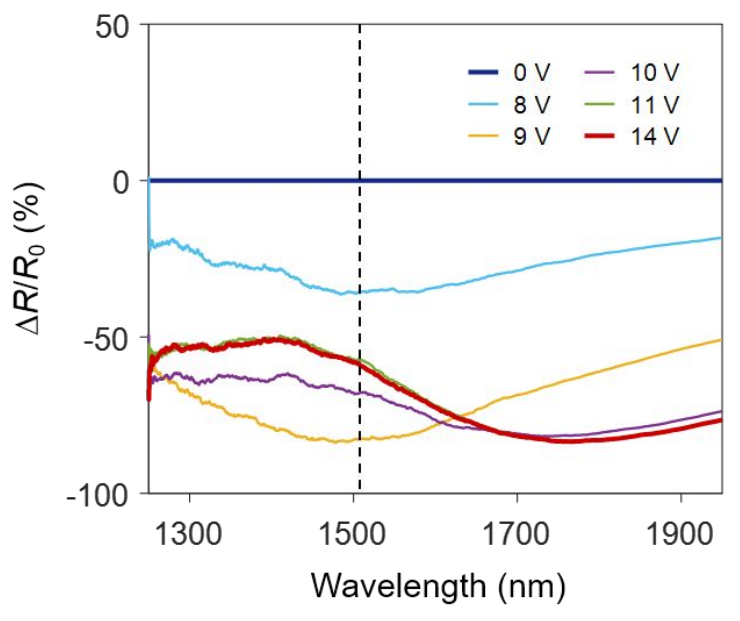

d

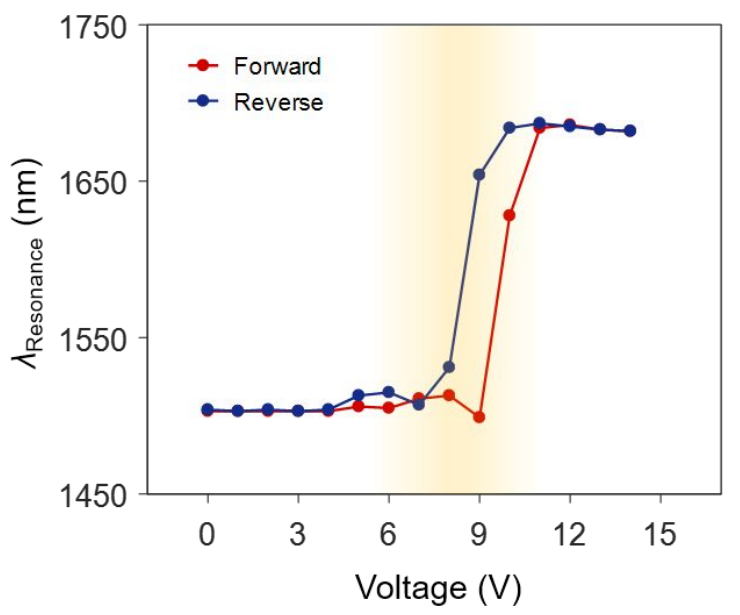


Figure 3. Experimental results of amplitude modulation. (a) Reflectance spectra and (b) relative reflectance change for different applied biases. The dashed line in Figure (b) indicates the insulating phase resonance wavelength observed in Figure (a) at a wavelength of $\lambda=1505 \mathrm{~nm}$. (c) Hysteresis loop in reflectance modulation as a function of applied bias. The reflectance is extracted at $\lambda=1550 \mathrm{~nm}$. (d) Hysteretic behavior in voltage-dependent resonance wavelength of the reflectance spectra. The hysteresis loops are reversible upon electrical bias cycles. The phase transition in $\mathrm{VO}_{2}$ is induced by applying electrical biases on the top metal stripe for Joule heating.

In order to characterize the actively tunable optical response of the $\mathrm{VO}_{2}$ metasurface, we used a Fourier transform infrared (FTIR) microscope to collect the reflectance spectra $(R)$ for different electrical biases applied to the top metal stripes (Figure $3 \mathrm{a}$ ). The absorbance $(A)$ of the metasurface can be obtained from the relation $A=1-R-T$, where the transmittance $(T)$ is negligible in the wavelength regime because of an optically-thick Au backplane. The performance of the metasurface measured by the FTIR is in good agreement with the full-wave simulation results (Figure 2c). When the $\mathrm{VO}_{2}$ metasurface is in the insulating phase with zero-bias, we experimentally observe a resonance dip at a wavelength of $1505 \mathrm{~nm}$. A gradual decrease in the resonance dip is observed as the applied bias increases up to $9 \mathrm{~V}$. From the simulation we expect almost unity absorption at an intermediate state $\mathrm{R}(0.6)$, while the measured data show slightly lower absorption (96.5\%) at a resonance wavelength of $\lambda=1505 \mathrm{~nm}$. Our experimental results show that the position of the resonance dip redshifts to approximately $1680 \mathrm{~nm}$ as $\mathrm{VO}_{2}$ is fully switched to the rutile phase with a peak absorption of $94.1 \%$. As compared to our simulation results, the fabricated device exhibits a larger shift of the resonance dip position by $175 \mathrm{~nm}$ and a smaller reflectance modulation $(17.2 \%$ at $\lambda=1505 \mathrm{~nm}$ and $23.5 \%$ at $\lambda=1680 \mathrm{~nm})$. In terms of modulation depth of electrically tunable $\mathrm{VO}_{2}$ metasurfaces, a thin-film-based metasurface showed a reflectance tuning of $80 \%$ in the mid-infrared, ${ }^{42}$ while an absorption modulation of $33 \%$ was achieved in the near-infrared by utilizing $\mathrm{VO}_{2}$ nanostructures located in the gap of the bow-tie antenna structures. $^{43}$

Figure $3 \mathrm{~b}$ illustrates the measured relative reflectance change $\Delta R / R_{0}=\left[R\left(V_{\mathrm{a}}\right)-R\left(V_{0}\right)\right] /$ $R\left(V_{0}\right)$, where $R\left(V_{0}\right)$ is the reflectance at zero-bias (applied bias $V_{\mathrm{a}}=0$ ) and $\Delta R$ is the difference between the reflectance at the applied bias value and zero-bias. We observe a large change in relative reflectance at the resonant dip wavelengths, which correspond to the cases of purely insulating and purely metallic phases of $\mathrm{VO}_{2}$. In case of the purely insulating phase, the relative reflectance modulation is $-80 \%$, while in case of the purely metallic phase, the relative reflectance 
modulation is $-78 \%$. We note that reflectance at $\lambda=1505 \mathrm{~nm}$ (insulating phase resonance dip position) exhibits a non-monotonic behavior under applied bias. This is because of the large redshift of the resonance dip when the applied bias is larger than $9 \mathrm{~V}$. On the other hand, the reflectance at $\lambda=1680 \mathrm{~nm}$ (metallic phase resonance dip position) exhibits monotonic change under applied bias.

Next, we plot the measured reflectance at a wavelength of $\lambda=1550 \mathrm{~nm}$ for cyclicallyapplied electrical bias ( $V_{\mathrm{a}}$ is first gradually increased from 0 to $14 \mathrm{~V}$, and then $V_{\mathrm{a}}$ is gradually decreased from 14 to $0 \mathrm{~V}$ ). We observe that the optical modulation is more pronounced when the applied voltage $V_{\mathrm{a}}$ exceeds $5 \mathrm{~V}$, which corresponds to the phase transition threshold. As seen in Figure $3 \mathrm{c}$, the reflectance at $\lambda=1550 \mathrm{~nm}$ reaches its minimum value at $V_{\mathrm{a}}=9 \mathrm{~V}$ and saturates when the applied voltage $V_{\mathrm{a}}$ exceeds $11 \mathrm{~V}$, which implies that $\mathrm{VO}_{2}$ layer is completely in the rutile phase. The switching power for the complete phase transition in $\mathrm{VO}_{2}$ was found to be $151.8 \mathrm{~mW}$, which yields an efficiency of $0.6 \mathrm{~mW}$ per antenna element. Furthermore, a hysteretic behavior is seen in the cycle of forward and reverse switching. The slope of the voltage-dependent reflectance is the steepest in the hysteretic region, where both insulating and metallic $\mathrm{VO}_{2}$ phases coexist. In addition, we use the results shown in Figure $3 \mathrm{a}$ to extract the spectral position of the resonance dip as a function of applied voltage (see Figure 3d). As seen in Figure 3d, as the bias voltage increases the resonance dip undergoes a slight blueshift immediately before the drastic redshift. 
a

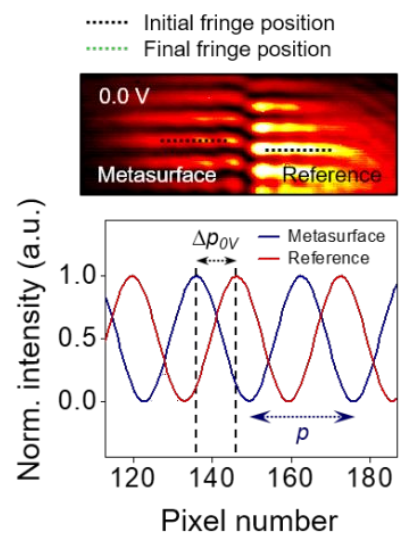

C

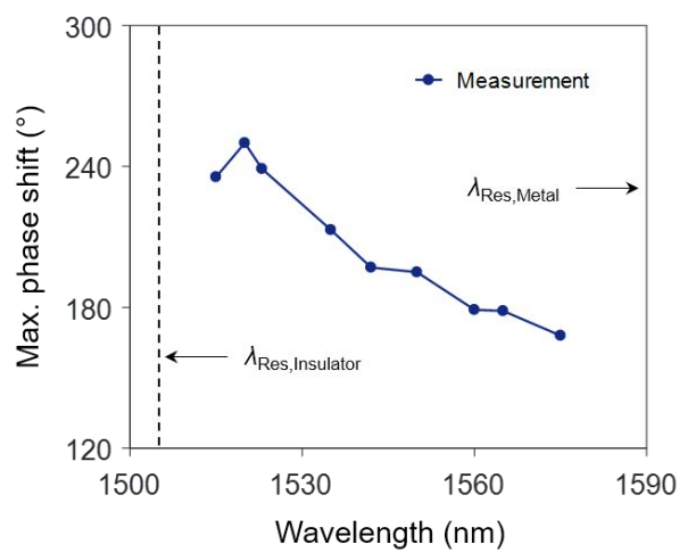

b
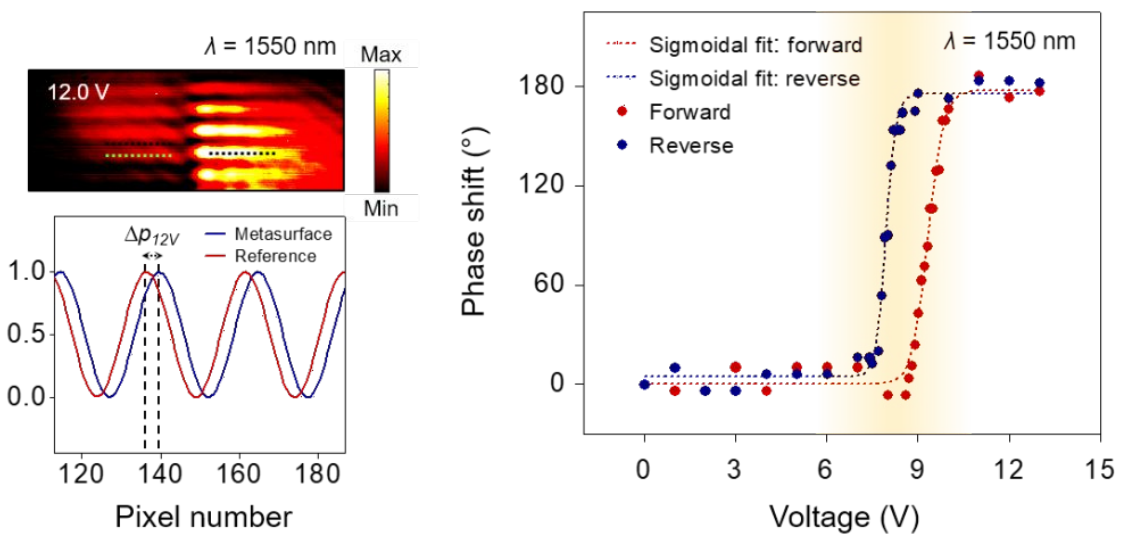

d

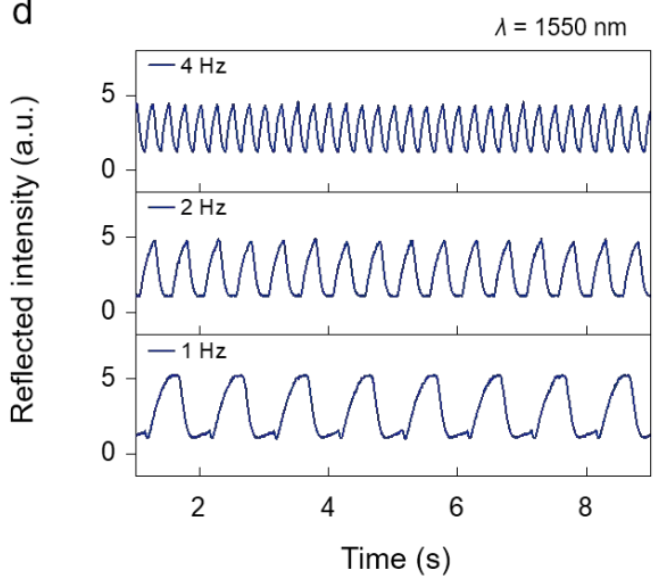

Figure 4. Experimental results of phase modulation and amplitude modulation speed. (a) Interference fringes for applied bias voltages of 0 and $12 \mathrm{~V}$ at a wavelength of $\lambda=1550 \mathrm{~nm}$. The dashed black lines indicate initial fringe positions while the dashed green line shows the displacement of the metasurface fringe when $V_{\mathrm{a}}=12 \mathrm{~V}$. (b) Continuously tunable phase shift of the metasurface as a function of applied voltage between 0 and $13 \mathrm{~V}$. It shows a reversible voltagedependent hysteresis loop in the phase shift. (c) Maximal achievable phase shift as a function of wavelength. A larger maximal phase shift can be obtained when the working wavelength is close to the insulating phase resonance dip. (d) Temporal response of the metasurface measured at a wavelength of $\lambda=1550 \mathrm{~nm}$.

As we have seen in our simulation results (Figure 2d), our metasurface not only controls the reflectance but also provides a platform to continuously tune the phase of the reflected light. In order to experimentally characterize the phase response of the $\mathrm{VO}_{2}$ metasurface, we used a Michelson-type interferometer setup (Supporting Information, Part 6). In our measurements, the incident laser beam is aligned to the edge of the metasurface structures so that one half of the beam illuminates the metasurface while the other half is reflected from the planar $\mathrm{Au} / \mathrm{Al}_{2} \mathrm{O}_{3}$ 
heterostructure. This approach enables us to use the planar $\mathrm{Au} / \mathrm{Al}_{2} \mathrm{O}_{3}$ heterostructure as a phase reference. The images of the interference fringes from both positions were simultaneously collected by a charge-coupled-device camera. This experimental setup configuration enables the measurement of the relative phase between the beam reflected from the metasurface and the reference beam. The top images of Figure 4a show the clear interference fringes at different biases that were measured at a wavelength of $\lambda=1550 \mathrm{~nm}$. The interference fringes from the metasurface structures (left-hand side) shifted downward as the applied bias is increased from 0 to $12 \mathrm{~V}$. We extracted the cross-sectional profiles of the interference fringes and fitted them to sinusoids. This enabled us to calculate a phase shift as a function of applied bias (bottom images of Figure 4a). ${ }^{19,20}$

Figure $4 \mathrm{~b}$ shows the phase shift result as a function of applied bias measured at $\lambda=$ $1550 \mathrm{~nm}$. The applied bias is gradually increased from 0 to $13 \mathrm{~V}$. This enables us to finely tune the dielectric permittivity of the $\mathrm{VO}_{2}$ layer via phase change and study an actively tunable phase response of the metasurface. In case of forward switching (when the applied bias is increased from 0 to $13 \mathrm{~V}$ ), the phase begins to experience significant variation when the voltage value exceeds $\approx 9 \mathrm{~V}$. For in-between values of $9 \mathrm{~V} \leq V_{\mathrm{a}} \leq 11 \mathrm{~V}$, a continuous phase shift is observed with a maximal achievable phase shift $>180^{\circ}$. Similar to the case of the voltage-dependent reflectance measurement (Figure 3c) and resonance dip position analysis (Figure 3d), we also observe a hysteresis loop when performing our phase shift measurements within a modulation cycle. It is noteworthy that the hysteresis of the voltage-dependent phase shift is in a good agreement with that of the voltage-dependent resonance dip position analysis. This result implies that the observed large phase modulation of the reflected light can be attributed to the large modulation of the resonance characteristics of the metasurface induced by the effective dielectric permittivity tuning of the $\mathrm{VO}_{2}$ layer. As we have seen in Figure 2a,c, the region that shows a large redshift in the resonance dip corresponds to the ENZ region in which the real part of the dielectric permittivity of $\mathrm{VO}_{2}$ in the resonant cavity changes sign from positive to negative.

To further emphasize the utility of our metasurface device, the maximal achievable phase shifts as a function of wavelength are presented in Figure 4c. A larger phase shift can be achieved when the wavelength is near the insulating phase resonance wavelength $(\lambda=1505 \mathrm{~nm})$. This tendency is consistent with full-wave simulation results as shown in Figure 2d. It is noteworthy that the results show a wide wavelength range that exhibits a phase shift $>180^{\circ}$, which implies that the device is not sensitive to the working wavelength, contributing to the flexibility of the 
device for practical applications. Furthermore, at an operating wavelength of $1520 \mathrm{~nm}$, the largest phase shift of $\approx 250^{\circ}$ is achieved upon the complete phase transition of the $\mathrm{VO}_{2}$ layer, which is slightly smaller than the simulation result. The deviation from the simulation is primarily due to sample inhomogeneity and index mismatch. For example, the structural inhomogeneity over the width of the antenna can induce variation in resonance characteristics of reflected light as we observed in Figure 2c (simulation) and Figure $3 a$ (measurement).

The response time measurements of the fabricated device are shown in Figure 4d. To characterize the frequency response of the metasurface, $11 \mathrm{~V}_{\mathrm{pp}}$ rectangular voltage pulses with a $50 \%$ duty cycle were applied to the device. The electrical bias value was chosen because it represents the reflectance-saturation bias value for the forward switching case as observed in Figure 3c. A high-speed InGaAs detector was used to detect the reflectance from the metasurface at a wavelength of $\lambda=1550 \mathrm{~nm}$ (Supporting Information, Part 7). As the modulation frequency increases above $2 \mathrm{~Hz}$, the amplitude of the modulated signal decreases, which implies that the $\mathrm{VO}_{2}$ does not completely change phase at these rates. The measured $\mathrm{ON}\left(V_{\mathrm{a}}=11 \mathrm{~V}\right)$ and $\mathrm{OFF}\left(V_{\mathrm{a}}=\right.$ $0 \mathrm{~V}$ ) switching time for the $1 \mathrm{~Hz}$ pulses were $\approx 500 \mathrm{~ms}$ and $\approx 250 \mathrm{~ms}$, respectively. However, the switching speed is by no means an intrinsic limit of the material. Previously, the ON and OFF switching speeds of less than $1 \mu \mathrm{s}$ and a few microseconds, respectively, were observed by reducing the heat capacity of the devices, ${ }^{43}$ for example, by decreasing the active region $\left(\mathrm{VO}_{2}\right)$ of the device. Furthermore, the ON and OFF switching speeds can be enhanced by applying highintensity short electrical pulses that enable localized Joule heating rather than global Joule heating as observed in the previous reports. ${ }^{42}$ The measured reflectance modulation speed indicates the $\mathrm{ON}$ and OFF switching times of $\approx 15 \mathrm{~ms}$ and $\approx 100 \mathrm{~ms}$, respectively (Supporting Information, Part 7). It is anticipated that the OFF switching response can be further improved by reducing the heat capacity of the device, for example, by decreasing the device size or active region $\left(\mathrm{VO}_{2}\right)$ of the device $^{43}$ and also by introducing a two-terminal type device that directly heats an active region only. ${ }^{62}$

In summary, we have demonstrated for the first time electrically-tunable continuous phase modulation of reflected infrared light in our phase-change-material-based metasurfaces. The tunable phase response is achieved via active control of the $\mathrm{VO}_{2}$ effective dielectric permittivity by Joule heating. We observe a maximal achievable phase shift up to $250^{\circ}$ over a wide wavelength range near the zero-bias resonance frequency. Hysteresis curves are observed in voltage-dependent 
reflectance, resonance wavelength, and phase shift analyses, which could be useful in electrically rewritable memory devices. We also report the response time of our $\mathrm{VO}_{2}$-based metasurface as $\approx 15 \mathrm{~ms}$ for ON switching and $\approx 100 \mathrm{~ms}$ for OFF switching when high-intensity short pulses are applied, which could be improved via optimizing the thermal engineering of the device. These characteristics demonstrate that electrically tunable phase-change metasurfaces have potential in versatile applications such as beam steering, reconfigurable lenses, and holographic imaging, where dynamical manipulation of light is required. 


\section{ASSOCIATED CONTENT \\ Supporting information}

Additional explanation for device fabrication, thin film characterization, full-wave simulation, effective medium approximation, optical setups, and supplementary temporal response measurement results

\section{AUTHOR INFORMATION \\ Corresponding Author}

*E-mail: haa@caltech.edu

\section{ORCID}

Yonghwi Kim: 0000-0002-6652-7994

Pin Chieh Wu: 0000-0002-5781-9696

Ruzan Sokhoyan: 0000-0003-4599-6350

Harry A. Atwater: 0000-0001-9435-0201

\section{Notes}

The authors declare no competing financial interest.

\section{ACKNOWLEDGEMENTS}

This work was supported by Samsung Electronics (Y.K., P.C.W., R.S., G.K.S.), the Ministry of Science and Technology, Taiwan under Grant 106-2917-I-564-049 (P.C.W.), and the Office of Science, U.S. Department of Energy (DOE) Office of Science Grant DE-FG02-07ER46405 (K.A.M. and H.A.A.). The authors used facilities supported by the Kavli Nanoscience Institute (KNI). Y.K. acknowledges the support from the Kwanjeong Educational Foundation scholarship. Y.K. also thanks J. Wong for help with the heating stage setup and G. Rossman for assistance with the initial FTIR measurements.

\section{REFERENCES}

(1) Yu, N.; Genevet, P.; Kats, M. A.; Aieta, F.; Tetienne, J.-P.; Capasso, F.; Gaburro, Z. Science 2011, 334 (6054), 333-337.

(2) Kildishev, A. V.; Boltasseva, A.; Shalaev, V. M. Science 2013, 339 (6125), 1232009.

(3) Arbabi, A.; Horie, Y.; Bagheri, M.; Faraon, A. Nat. Nanotechnol. 2015, 10 (11), 937-943.

(4) Sun, S.; Yang, K.-Y.; Wang, C.-M.; Juan, T.-K.; Chen, W. T.; Liao, C. Y.; He, Q.; Xiao, S.; Kung, W.-T.; Guo, G.-Y.; et al. Nano Lett. 2012, 12 (12), 6223-6229.

(5) Pors, A.; Albrektsen, O.; Radko, I. P.; Bozhevolnyi, S. I. Sci. Rep. 2013, 3, 2155.

(6) Lin, D.; Fan, P.; Hasman, E.; Brongersma, M. L. Science 2014, 345 (6194), 298-302.

(7) Khorasaninejad, M.; Chen, W. T.; Devlin, R. C.; Oh, J.; Zhu, A. Y.; Capasso, F. Science 2016, 352 (6290), 1190-1194.

(8) Wu, P. C.; Tsai, W.-Y.; Chen, W. T.; Huang, Y.-W.; Chen, T.-Y.; Chen, J.-W.; Liao, C. Y.; Chu, C. H.; Sun, G.; Tsai, D. P. Nano Lett. 2017, 17 (1), 445-452.

(9) Black, L.-J.; Wang, Y.; de Groot, C. H.; Arbouet, A.; Muskens, O. L. ACS Nano 2014, 8 (6), 6390-6399.

(10) Chen, W. T.; Yang, K.-Y.; Wang, C.-M.; Huang, Y.-W.; Sun, G.; Chiang, I.-D.; Liao, C. Y.; Hsu, W.-L.; Lin, H. T.; Sun, S.; et al. Nano Lett. 2014, 14 (1), 225-230. 
(11) Zheng, G.; Mühlenbernd, H.; Kenney, M.; Li, G.; Zentgraf, T.; Zhang, S. Nat. Nanotechnol. 2015, 10 (4), 308-312.

(12) Huang, Y.-W.; Chen, W. T.; Tsai, W.-Y.; Wu, P. C.; Wang, C.-M.; Sun, G.; Tsai, D. P. Nano Lett. 2015, 15 (5), 3122-3127.

(13) Lewi, T.; Evans, H. A.; Butakov, N. A.; Schuller, J. A. Nano Lett. 2017, 17 (6), 3940-3945.

(14) Rahmani, M.; Xu, L.; Miroshnichenko, A. E.; Komar, A.; Camacho-Morales, R.; Chen, H.; Zárate, Y.; Kruk, S.; Zhang, G.; Neshev, D. N.; et al. Adv. Funct. Mater. 2017, 27 (31), 1700580.

(15) Horie, Y.; Arbabi, A.; Arbabi, E.; Kamali, S. M.; Faraon, A. ACS Photonics 2018, 5 (5), 17111717.

(16) Yi, F.; Shim, E.; Zhu, A. Y.; Zhu, H.; Reed, J. C.; Cubukcu, E. Appl. Phys. Lett. 2013, 102 (22), 221102.

(17) Park, J.; Kang, J.-H.; Liu, X.; Brongersma, M. L. Sci. Rep. 2015, 5, 15754.

(18) Park, J.; Kang, J.-H.; Kim, S. J.; Liu, X.; Brongersma, M. L. Nano Lett. 2017, 17 (1), 407-413.

(19) Huang, Y.-W.; Lee, H. W. H.; Sokhoyan, R.; Pala, R. A.; Thyagarajan, K.; Han, S.; Tsai, D. P.; Atwater, H. A. Nano Lett. 2016, 16 (9), 5319-5325.

(20) Kafaie Shirmanesh, G.; Sokhoyan, R.; Pala, R. A.; Atwater, H. A. Nano Lett. 2018, 18 (5), 2957-2963.

(21) Jun, Y. C.; Reno, J.; Ribaudo, T.; Shaner, E.; Greffet, J.-J.; Vassant, S.; Marquier, F.; Sinclair, M.; Brener, I. Nano Lett. 2013, 13 (11), 5391-5396.

(22) Olivieri, A.; Chen, C.; Hassan, S.; Lisicka-Skrzek, E.; Tait, R. N.; Berini, P. Nano Lett. 2015, 15 (4), 2304-2311.

(23) Yao, Y.; Kats, M. A.; Genevet, P.; Yu, N.; Song, Y.; Kong, J.; Capasso, F. Nano Lett. 2013, 13 (3), 1257-1264.

(24) Jang, M. S.; Brar, V. W.; Sherrott, M. C.; Lopez, J. J.; Kim, L.; Kim, S.; Choi, M.; Atwater, H. A. Phys. Rev. B 2014, 90 (16), 165409.

(25) Dabidian, N.; Kholmanov, I.; Khanikaev, A. B.; Tatar, K.; Trendafilov, S.; Mousavi, S. H.; Magnuson, C.; Ruoff, R. S.; Shvets, G. ACS Photonics 2015, 2 (2), 216-227.

(26) Wu, P. C.; Papasimakis, N.; Tsai, D. P. Phys. Rev. Appl. 2016, 6 (4), 044019.

(27) Sherrott, M. C.; Hon, P. W. C.; Fountaine, K. T.; Garcia, J. C.; Ponti, S. M.; Brar, V. W.; Sweatlock, L. A.; Atwater, H. A. Nano Lett. 2017, 17 (5), 3027-3034.

(28) Kim, S.; Jang, M. S.; Brar, V. W.; Mauser, K. W.; Kim, L.; Atwater, H. A. Nano Lett. 2018, 18 (2), 971-979.

(29) Chen, Y.; Li, X.; Sonnefraud, Y.; Fernández-Domínguez, A. I.; Luo, X.; Hong, M.; Maier, S. A. Sci. Rep. 2015, 5, 8660.

(30) Tittl, A.; Michel, A.-K. U.; Schäferling, M.; Yin, X.; Gholipour, B.; Cui, L.; Wuttig, M.; Taubner, T.; Neubrech, F.; Giessen, H. Adv. Mater. 2015, 27 (31), 4597-4603.

(31) Yin, X.; Steinle, T.; Huang, L.; Taubner, T.; Wuttig, M.; Zentgraf, T.; Giessen, H. Light Sci. Appl. 2017, 6 (7), e17016.

(32) Wang, Q.; Rogers, E. T. F.; Gholipour, B.; Wang, C.-M.; Yuan, G.; Teng, J.; Zheludev, N. I. Nat. Photonics 2016, 10 (1), 60-65.

(33) Galarreta, C. R. de; Alexeev, A. M.; Au, Y.-Y.; Lopez-Garcia, M.; Klemm, M.; Cryan, M.; Bertolotti, J.; Wright, C. D. Adv. Funct. Mater. 2018, 28 (10), 1704993.

(34) Hosseini, P.; Wright, C. D.; Bhaskaran, H. Nature 2014, 511 (7508), 206-211.

(35) Driscoll, T.; Palit, S.; Qazilbash, M. M.; Brehm, M.; Keilmann, F.; Chae, B.-G.; Yun, S.-J.; Kim, H.-T.; Cho, S. Y.; Jokerst, N. M.; et al. Appl. Phys. Lett. 2008, 93 (2), 024101.

(36) Dicken, M. J.; Aydin, K.; Pryce, I. M.; Sweatlock, L. A.; Boyd, E. M.; Walavalkar, S.; Ma, J.; Atwater, H. A. Opt. Express 2009, 17 (20), 18330-18339.

(37) Kats, M. A.; Sharma, D.; Lin, J.; Genevet, P.; Blanchard, R.; Yang, Z.; Qazilbash, M. M.; Basov, D. N.; Ramanathan, S.; Capasso, F. Appl. Phys. Lett. 2012, 101 (22), 221101. 
(38) Kocer, H.; Butun, S.; Banar, B.; Wang, K.; Tongay, S.; Wu, J.; Aydin, K. Appl. Phys. Lett. 2015, 106 (16), 161104.

(39) Dong, K.; Hong, S.; Deng, Y.; Ma, H.; Li, J.; Wang, X.; Yeo, J.; Wang, L.; Lou, S.; Tom, K. B.; et al. Adv. Mater. 2018, 30 (5), 1703878.

(40) Liu, M.; Hwang, H. Y.; Tao, H.; Strikwerda, A. C.; Fan, K.; Keiser, G. R.; Sternbach, A. J.; West, K. G.; Kittiwatanakul, S.; Lu, J.; et al. Nature 2012, 487 (7407), 345-348.

(41) Driscoll, T.; Kim, H.-T.; Chae, B.-G.; Kim, B.-J.; Lee, Y.-W.; Jokerst, N. M.; Palit, S.; Smith, D. R.; Ventra, M. D.; Basov, D. N. Science 2009, 325 (5947), 1518-1521.

(42) Liu, L.; Kang, L.; Mayer, T. S.; Werner, D. H. Nat. Commun. 2016, 7, 13236.

(43) Zhu, Z.; Evans, P. G.; Haglund, R. F.; Valentine, J. G. Nano Lett. 2017, 17 (8), 4881-4885.

(44) Hashemi, M. R. M.; Yang, S.-H.; Wang, T.; Sepúlveda, N.; Jarrahi, M. Sci. Rep. 2016, 6, 35439.

(45) Decker, M.; Kremers, C.; Minovich, A.; Staude, I.; Miroshnichenko, A. E.; Chigrin, D.; Neshev, D. N.; Jagadish, C.; Kivshar, Y. S. Opt. Express 2013, 21 (7), 8879-8885.

(46) Sautter, J.; Staude, I.; Decker, M.; Rusak, E.; Neshev, D. N.; Brener, I.; Kivshar, Y. S. ACS Nano 2015, 9 (4), 4308-4315.

(47) Komar, A.; Paniagua-Domínguez, R.; Miroshnichenko, A.; Yu, Y. F.; Kivshar, Y. S.; Kuznetsov, A. I.; Neshev, D. ACS Photonics 2018, 5 (5), 1742-1748.

(48) Bohn, J.; Bucher, T.; Chong, K. E.; Komar, A.; Choi, D.-Y.; Neshev, D. N.; Kivshar, Y. S.; Pertsch, T.; Staude, I. Nano Lett. 2018, 18 (6), 3461-3465.

(49) Thyagarajan, K.; Sokhoyan, R.; Zornberg, L.; Atwater, H. A. Adv. Mater. 2017, 29 (31), 1701044.

(50) Ou, J.-Y.; Plum, E.; Zhang, J.; Zheludev, N. I. Nat. Nanotechnol. 2013, 8 (4), 252-255.

(51) Valente, J.; Ou, J.-Y.; Plum, E.; Youngs, I. J.; Zheludev, N. I. Nat. Commun. 2015, 6, 7021.

(52) Ee, H.-S.; Agarwal, R. Nano Lett. 2016, 16 (4), 2818-2823.

(53) Imada, M.; Fujimori, A.; Tokura, Y. Rev. Mod. Phys. 1998, 70 (4), 1039-1263.

(54) Qazilbash, M. M.; Brehm, M.; Chae, B.-G.; Ho, P.-C.; Andreev, G. O.; Kim, B.-J.; Yun, S. J.; Balatsky, A. V.; Maple, M. B.; Keilmann, F.; et al. Science 2007, 318 (5857), 1750-1753.

(55) Suh, J. Y.; Lopez, R.; Feldman, L. C.; Haglund, R. F. J. Appl. Phys. 2004, 96 (2), 1209-1213.

(56) Marvel, R. E.; Harl, R. R.; Craciun, V.; Rogers, B. R.; Haglund, R. F. Acta Mater. 2015, 91, 217-226.

(57) Rozen, J.; Lopez, R.; Haglund, R. F.; Feldman, L. C. Appl. Phys. Lett. 2006, 88 (8), 081902.

(58) Sharoni, A.; Ramírez, J. G.; Schuller, I. K. Phys. Rev. Lett. 2008, 101 (2), 026404.

(59) Choi, H. S.; Ahn, J. S.; Jung, J. H.; Noh, T. W.; Kim, D. H. Phys. Rev. B 1996, 54 (7), 46214628.

(60) Carr, G. L.; Perkowitz, S.; Tanner, D. B. Far-Infrared Properties of Inhomogeneous Materials. In Infrared and millimeter waves; Academic Press, 1985; Vol. 13, pp 171-263.

(61) Homes, C. C.; Xu, Z. J.; Wen, J. S.; Gu, G. D. Phys. Rev. B 2012, 86 (14), 144530.

(62) Markov, P.; Marvel, R. E.; Conley, H. J.; Miller, K. J.; Haglund, R. F.; Weiss, S. M. ACS Photonics 2015, 2 (8), 1175-1182. 


\section{TOC figure}

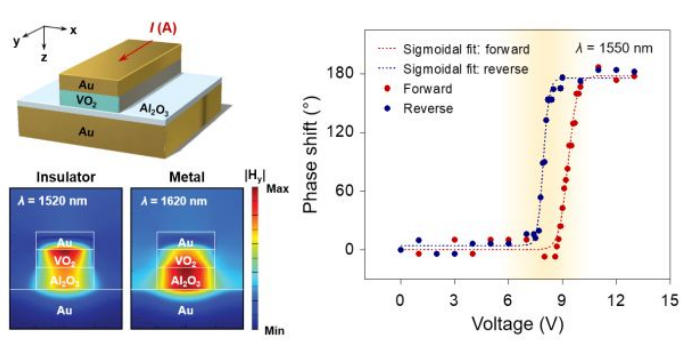

